\title{
Heart rate and heart rate variability in patients with chronic inflammatory joint disease: The role of pain duration and the insular cortex
}

\author{
Leona Katharin Buschmann ${ }^{1,2 凶}$, Melanie Spindler ${ }^{3}$, Peter Sörö̈s ${ }^{4,5,}$, and Carsten Bantel ${ }^{2,5, *}$ \\ ${ }^{1}$ Department of Anesthesiology, Intensive Care Medicine and Pain Therapy, Evangelisches Krankenhaus Oldenburg, Oldenburg, Germany \\ ${ }^{2}$ Anesthesiology, Critical Care, Emergency Medicine, and Pain Management, School of Medicine and Health Sciences, University of Oldenburg, Oldenburg, Germany \\ ${ }^{3}$ Biological Psychology, Department of Psychology, School of Medicine and Health Sciences, University of Oldenburg, Oldenburg, Germany \\ ${ }^{4}$ Neurology, School of Medicine and Health Sciences, University of Oldenburg, Oldenburg, Germany \\ ${ }^{5}$ Research Center Neurosensory Science, University of Oldenburg, Oldenburg, Germany \\ "These authors share senior authorship.
}

\begin{abstract}
Chronic inflammatory joint diseases (CIJD) have been linked to increased cardiovascular morbidity and mortality. A decisive reason could be a dysregulation of the autonomic nervous system (ANS), which is responsible for the control of cardiovascular function. So far, the cause of changes in ANS functions remains elusive. In this study, we investigate the role of chronic pain and the insular cortex in autonomic control of cardiac functioning in patients with CIJD. We studied the sympathetic and parasympathetic branch of the ANS through the assessment of heart rate and heart rate variability (HRV) at rest and under cognitive stimulation. Furthermore, we investigated insular cortex volume by performing surface-based brain morphometry with FreeSurfer. For this study, 22 individual age- and sex-matched pairs for the magnetic resonance imaging analyses and 14 for the HRV analyses were recruited. Pain duration was negatively correlated with the resting heart rate in patients with chronic inflammatory joint diseases $(n=19)$. In a multiple linear regression model including only CIJD patients with HR at rest as a dependent variable, we found a significant positive relationship between $H R$ at rest and the volume of the left insular cortex and a significant negative relationship between HR at rest and the volume of the right insular cortex. However, we found no significant differences in HRV parameters or insular cortex volumes between both groups. In this study we provide evidence to suggest insular cortex involvement in the process of ANS changes due to chronic pain in CIJD patients.
\end{abstract}

Chronic inflammatory joint diseases | chronic pain | pain duration | insular cortex | heart rate | heart rate variability

Correspondence: Ik.buschmann@gmail.com

\section{Introduction}

Chronic inflammatory joint diseases (CIJD), such as rheumatoid arthritis (RA) and ankylosing spondylitis, are commonly associated with disability and pain [37]. CIJDs have also been linked to increased cardiovascular morbidity and mortality, which cannot be attributed to typical risk factors, such as smoking or hypertension [14; 22; 44; 68; 81]. Instead, a dysregulation of the autonomic nervous system (ANS) has been suggested as a potential underlying mechanism. This notion is based on an estimate of approximately $60 \%$ of patients with RA showing signs of ANS dysfunction [5].

The function of the ANS can be assessed by measuring heart rate and calculating heart rate variability (HRV), i.e. the vari- ation of the length of the R-to-R-wave time interval [34; 63]. In patients with rheumatic diseases, a reduction of HRV has been observed, indicating reduced cardiac adaptability [5;24; 36]. However, the reasons for these changes in ANS function in CIJD remain elusive. Based on the results of a study by Dunn and Croft from 2006, who showed the prognosis of low back pain was dependent on the duration of pain, it could be possible that changes in HRV might be associated with pain duration [12].

The ANS is controlled by centers in the brainstem, the hypothalamus, and, ultimately, by several cortical regions, including the insula, anterior cingulate gyrus, and medial frontal cortex $[9 ; 55]$. The insula is considered a crucial region for polymodal sensory, cognitive, and affective processing [23]. In addition to the processing function of somatosensory [72; 73], peripheral inflammatory [40], and nociceptive stimuli [29], the insular cortex participates in the control of arbitrary and involuntary movements, such as speech production [4] and swallowing [71]. Oppenheimer et al. were first to demonstrate changes in cardiac function after electrical stimulation of the left and right insula in humans [51]. Corroborating these results, several studies reported cardiac dysfunction following insula lesions due to stroke, tumors, or surgical resections $[16 ; 47 ; 65 ; 70]$.

Wartolowska and colleagues used surface-based and voxelbased morphometry as well as segmentations of subcortical nuclei to investigate morphological changes in the brains of patients with rheumatoid arthritis. This study found an increase in gray matter in the basal ganglia of the patient group, mainly in the nucleus accumbens and caudate nucleus, without significant differences in cortical gray matter [80]. Similarly to what has been described for other chronic pain conditions such as chronic back and limb pain $[8 ; 56]$, complex regional pain syndrome [30], migraine [58], chronic tension type-headache [59], and fibromyalgia [41], patients with CIJD might also display structural changes in cortical regions that are involved in the regulation of the ANS, especially in the insular cortex.

The aims of the present study hence were a) to evaluate differences in HRV at rest and under stimulation between the CIJD and control groups, b) to test whether pain duration might be associated with changes in heart rate and heart rate variabil-

NOTE: This preprint reports new research that has not been certified by peer review and should not be used to guide clinical practice. 
ity in patients with chronic inflammatory joint diseases, and c) whether altered HRV is associated with structural changes in ANS-controlling cortical areas such as the insular cortex.

\section{Methods}

The study was approved by the Medical Research Ethics Board of Carl von Ossietzky University of Oldenburg, Germany (\#2017-059) and was preregistered with the German Clinical Trials Register (https://www.drks.de; DRKS00012791). All participants gave written informed consent prior to participating in this study. Participants (healthy controls: C, patients with chronic inflammatory joint diseases: CIJD) were recruited between July 2017 and March 2019. All measurements were performed at the Neuroimaging Unit, School of Medicine and Health Sciences, Carl von Ossietzky University of Oldenburg. All data were collected as a part of a larger study, which, in addition to the data evaluated here, included functional magnetic resonance imaging (fMRI) and behavioral tests [69; 74].

Participants. Patients aged between 18 and 75 years were recruited at a specialized rheumatological outpatient clinic and with the help of a support group for ankylosing spondylitis patients in Oldenburg. Controls were recruited by advertisements in a local newspaper and announcements on the university's website. Participants had to be fluent in German and right-handed. Patients needed to suffer from a chronic inflammatory joint disease that caused chronic pain for at least one year while controls had to be free of CIJD and chronic pain in general. Controls and CIJD were matched for sex and age ( \pm 5 years).

Pregnant women and participants with contraindications for MRI were excluded, in addition to participants with conditions that could have had an influence on HRV or cerebral functions: previous heart surgeries; heart failure stage $>2$ of the New York Heart Association's classification [53]; peripheral artery diseases type 2 - 4 according to Fontaine [3]; neurological diseases (e.g. stroke, multiple sclerosis, myasthenia gravis, known cerebrovascular disease); active cancer; chronic liver diseases (hepatitis or liver failure); renal failure requiring dialysis; severe asthma and severe chronic obstructive pulmonary disease (COPD); poorly adjusted thyroid diseases; infections (tuberculosis, human immunodeficiency virus); psychiatric disorders (schizophrenia, severe depression, borderline personality disorder) and dyscalculia. Finally, patients with medications that might have influenced the conduction system of the heart or/and active or previous alcohol or drug abuse were also excluded.

Experimental procedure. First, each participant completed a questionnaire including information about demographic and clinical characteristics, such as pain, co-morbidities, and suitability for MRI-examinations. Next, structural and functional MRI was performed while the measurements of HRV at rest and under cognitive stimulation were carried out.

Data acquisition. Information about the diagnosis, pain intensity, duration of pain, and medications were collected in the CIJD group. Pain intensity was assessed using an 11point numerical rating scale (NRS, $0=$ no pain and $10=$ worst pain imaginable) [12]. Photoplethysmographic pulse oximetry was used to determine HRV. Data recording was performed using the integrated Siemens Physiological Monitoring Unit of the body scanner (MAGNETOM Prisma, Siemens, Erlangen, Germany). In short, an infrared emitter was placed on the left index finger. The pulse curve was recorded at $50 \mathrm{~Hz}$ and heart rates, as well as the length of the intervals between heartbeats, were determined. All signals were transmitted wirelessly to the scanner and saved for later analysis.

MR images were acquired by a research-dedicated 3T wholebody scanner (MAGNETOM Prisma, Siemens, Erlangen, Germany) with a 64-channel head/neck coil. T1-weighted images were obtained with a magnetization prepared rapid gradient echo sequence (MP-RAGE) with the following parameters: voxel size: $0.75 \times 0.75 \times 0.75 \mathrm{~mm}, 224$ sagittal slices, repetition time (TR): $2000 \mathrm{~ms}$, echo time (TE): 2.07 $\mathrm{ms}$, inversion time (TI): $952 \mathrm{~ms}$, anterior to posterior phase encoding direction, flip-angle: $9^{\circ}$, in-plane acceleration with an acceleration factor of 2 (GRAPPA), and an acquisition time of 6:16 minutes.

Experimental paradigm. Three functional MRI experiments were performed: an arithmetic experiment with two subtraction tasks, a number line identification experiment, and a resting-state measurement. Here, the HRV signals recorded during the arithmetic experiment and the restingstate measurement were analyzed as a marker of autonomic function [76]. The analysis of task-based and resting-state fMRI data will be presented in another publication.

As in the Trier social stress test, serial subtraction was employed to stimulate a cardiac response [39]. The arithmetic experiment consisted of four conditions: (1) simple serial subtraction (e.g., starting with 99 - 7), (2) difficult serial subtraction (e.g., starting with 173-13), (3) continuously reading aloud a number presented on the screen, and (4) a rest condition, fixating a cross on the screen. Every condition lasted $20 \mathrm{~s}$ and was repeated with different numbers five times during the experiment. For the subtraction conditions, the participants were instructed to perform serial subtractions as quickly as possible and say out loud every result. Finally, after a short break of less than a minute, a resting state measurement took place. Short breaks between the tasks were sufficient, as the HRV should return rapidly to a normal level after temporary stimulation [1].

Coupling MRI tasks and pulse oximetry measurements. The Physiological Artifact Removal Tool (PART, https://www.mccauslandcenter.sc.edu/crnl/tools/part) was used to link the starting points of the MRI tasks and pulse oximetry measurements. The MRI measurements included 205 volumes each in the arithmetic task and resting state. For the analysis of the pulse oximetry measurements, the first 4 volumes were deleted. This led to a duration of 6 minutes and 42 seconds for each HRV measurement. 
Analysis of heart rate variability. Data processing and calculation of the HRV via pulse oximetry measurements were performed with Kubios HRV standard (Version 3.2.0, http://www.kubios.com/), which is an established software for assessing ANS function [75]. Artifacts such as extra, missing, or ectopic beats can cause considerable distortion of HRV results [11]. Therefore, 'threshold-based correction' was employed and preset to 'low'. The correction algorithm detected and corrected every beat-to-beat interval that was 0.35 seconds longer or shorter compared to the local average calculated using an internal software algorithm. Corrected data were divided into time and frequency domain parameters. Time-domain parameters included the heart rate (HR) in beats per minute (bpm), and the standard deviation of all normal-to-normal beat intervals (SDNN) in ms, which reflects the variation within the $\mathrm{R}$-waves intervals. The frequency data were divided into low frequency (LF) and high frequency (HF) bands. The absolute power of the different frequency bands was specified in $\mathrm{ms}^{2} / \mathrm{Hz}$. The frequency ranges for the absolute power measurements were as follows: $\mathrm{LF}=0.04-0.15 \mathrm{~Hz}$ and $\mathrm{HF}=0.15-0.4 \mathrm{~Hz}$ [75]. LF and $\mathrm{HF}$ were used to characterize the response of the ANS. The HF band is thought to reflect the activity of the cardiac parasympathetic function $[46 ; 63]$. The physiological meaning of the LF is controversial. Partly representations of the sympathetic and parasympathetic nervous system, separate sections of the parasympathetic branch of the ANS, or the oscillations of the baroreflex, are discussed [31; 54; 63]. HR, SDNN, LF, and $\mathrm{HF}$ are some of the most used parameters for HRV analysis [49;63].

Analysis of MR-images. The image analysis, performed with FreeSurfer (Version 6.0.0, released January 2017, http://surfer.nmr.mgh.harvard.edu) on Mac OS high sierra (Version 10.13.6), started with the extraction of the cortical (pial) surface. Due to the hybrid watershed/surface deformation procedure, non-brain tissues were removed [60]. Additionally, segmentation of gray and white matter and intensity normalization was performed $[26 ; 66]$. The surfaces were constructed as a grid of triangles, placed in areas with the greatest intensity shift. The intensity shift was caused by the transition between different tissues [20]. Next, the software modeled the surface between gray and white matter and the outer-pial surface, which represented the boundary of gray matter to cerebrospinal fluid. The final step was the automated topological correction to fix segmentation errors and produce a closed sphere [25; 61]. Regardless of the MRI scanner and field strength, the morphometric test-retest reliability is high [33]. The quality of the created surfaces and subcortical structures was visually checked for each participant.

Cortical volume analysis. FreeSurfer performed an automatic reconstruction of the brain's surface. The segmentation procedure and the parcellation of the cerebral cortex were one of the steps of the command recon-all, which included the extraction of the white matter and other structures, a vertex-wise reconstruction of the surfaces, smoothing, in- flation, and localization of topological defects. In the end, possible tracked defects were automatically fixed. The system used the probability of the localizations of subcortical structures [26; 27]. In the process of cortical parcellation, the units were assigned according to gyral and sulcal structures. Data evaluation was based on the atlas by Desikan et al. [21; 28]. The regions of interest (ROI) for this study were the insulae as processing centers of the ANS in the left and right hemispheres. The cortical brain data were normalized using the estimated total intracranial volume (eTIV) generated by FreeSurfer [13; 45].

Statistical Analysis. All statistical analyses were performed using SPSS 25 (IBM, Ehningen, Germany). Data were analyzed for normal distribution employing ShapiroWilk tests. SDNN, LF, and HF were logarithmically transformed to approximate normal distribution. Demographic data were first analyzed descriptively. To determine possible differences between the demographic data of the groups, independent samples t-tests and chi-square tests were used. Repeated measure analysis of variance (ANOVA) was employed to evaluate possible differences between the HRV parameters in the different measurements and between the groups. The effect size $\eta^{2}$ was calculated and interpreted according to Cohen $(<0.01=$ small, $0.06=$ medium and $>0.14=$ large effect $)$ [17].

Bivariate Pearson's correlation analyses between HRV at rest and under stimulation, together with the pain duration in years were performed. The obtained correlation coefficients were interpreted according Cohen as small $(\mathrm{r}=0.1)$, medium $(r=0.3)$, or large $(r=0.5)$ [17]. Independent samples t-tests of the left and right insular cortex volume were performed between groups. Furthermore, four linear regression models were used to test the effect of independent influences. SDNN and HR at rest were included in the first model as dependent variables while group, age, sex, and volume of the normalized insulae in both hemispheres were defined as independent variables. HR and SDNN at rest were selected for initial analysis. Finally, a second regression with only CIJD was computed with SDNN and HR at rest as dependent variables. The model corresponded to the regression model described above, only that the variable age was exchanged for pain duration and the variable group was deleted. Results were considered statistically significant if $\mathrm{p}<0.05$.

\section{Results}

Characteristics of participants. Figure 1 summarizes the recruitment process; 47 participants were initially recruited (23 controls, 24 CIJD patients). One CIJD patient was excluded due to limited compliance during the MRI acquisition and one control participant due to beta-blocker intake. Five control and four CIJD participants were excluded from HRV analysis because of incomplete pulse recordings or poor data quality. Participants were matched on an individual basis: For every CIJD patient, a control participant of the same sex and similar age ( \pm 5 years) was recruited. For 22 CIJD patients and 22 matched controls, MRI data were available. For 19 
medRxiv preprint doi: https://doi.org/10.1101/2021.01.14.21249826; this version posted January 25, 2021. The copyright holder for this preprint (which was not certified by peer review) is the author/funder, who has granted medRxiv a license to display the preprint in perpetuity. It is made available under a CC-BY-NC-ND 4.0 International license .

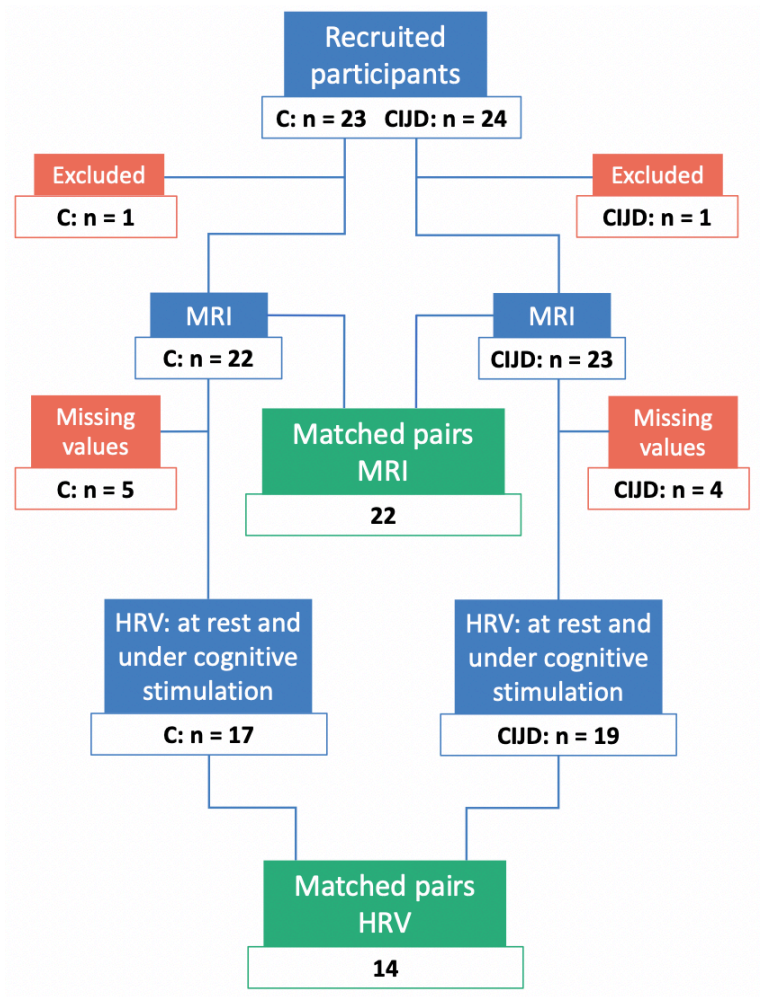

Fig. 1. Flow diagram of participant inclusion. The diagram illustrates the participant in- and exclusion process. Blue boxes: included participants; red boxes: excluded participants. Green boxes: sex and age ( \pm 5 years) matched pairs. Blue boxes with HRV-label: number of participants with HRV measurements under resting conditions and cognitive stimulation. C: controls; CIJD: patients with chronic inflammatory joint diseases; HRV: heart rate variability; MRI: magnetic resonance imaging

CIJD patients and 17 controls, complete pulse recordings at rest and under cognitive stimulation of sufficient quality were available, resulting in 14 matched pairs.

Table 1 summarizes the demographic and clinical characteristics of the patient and control groups. There were no significant differences between groups regarding age, smoking status, or formal education. No participants were taking opioids, benzodiazepines, or anticonvulsants at the time of the study. Seven CIJD patients were taking glucocorticoids at the time of the study; four CIJD patients had taken gluco-

Table 1. Characteristics of participants

\begin{tabular}{llll}
\hline & Controls & CIJD & p \\
\hline Sample size, $\mathrm{n}$ & 22 & 22 & \\
Gender (m/f) & $6 / 16$ & $6 / 16$ & \\
Age (years), mean (SD) & $50.9( \pm 10.6)$ & $51.3( \pm 10.2)$ & 0.896 \\
Smoking (yes/no/earlier) & $17 / 2 / 3$ & $14 / 3 / 5$ & 0.609 \\
Education* (median) & 3 & 2 & 0.550 \\
Chronic inflammatory joint diseases: & & & \\
- Rheumatoid arthritis; $\mathrm{n}$ & & 9 & \\
- Ankylosing spondylitis; $\mathrm{n}$ & & 9 & \\
- Psoriatic arthropathy; $\mathrm{n}$ & 3 & \\
- CREST syndrome**; $\mathrm{n}$ & 1 & \\
Pain duration (yrs), median (min/max) & & $10(1 / 44)$ & \\
Pain intensity, median (min/max) ${ }^{\star \star *}$ & & $3.5(0 / 7)$ & \\
\hline
\end{tabular}

SD: Standard deviation; CIJD: patients with chronic inflammatory joint diseases. * The level of education was classified according to the achieved academic degree: $0=$ no degree, 1 = secondary school, 2 = middle school, $3=$ High school diploma, 4 $=$ university/ college. ${ }^{* *}$ CREST $=$ calcinosis, Raynaud's phenomenon, esophageal motility abnormalities, sclerodactyly and telangiectasia. ${ }^{\star \star *} 11$-NRS $=11$-point numerical rating scale $(0=$ no pain to $10=$ worst pain imaginable), on the day of testing under usual medication
Table 2. Results of the four repeated measurements ANOVAS assessing the influence of the condition and group on HRV parameters

\begin{tabular}{lllll}
\hline & $\mathbf{F}$ & $\mathbf{d f}$ & $\mathbf{p}$ & $\eta^{2}$ \\
\hline Heart rate & & & & \\
Condition & 40.950 & 1,26 & $<0.001^{*}$ & 0.612 \\
Group & 0.182 & 1,26 & 0.673 & 0.007 \\
Interaction & 0.005 & 1,26 & 0.942 & $<0.001$ \\
SDNN & & & & \\
Condition & 12.090 & 1,26 & $0.002^{*}$ & 0.317 \\
Group & 0.377 & 1,26 & 0.545 & 0.014 \\
Interaction & 0.254 & 1,26 & 0.619 & 0.010 \\
Low frequency power & & & & \\
Condition & 10.362 & 1,26 & $0.003^{*}$ & 0.285 \\
Group & 0.237 & 1,26 & 0.631 & 0.009 \\
Interaction & 4.851 & 1,26 & $0.037^{*}$ & 0.157 \\
$\quad$ High frequency power & & & & \\
$\quad$ Condition & 3.140 & 1,26 & 0.088 & 0.108 \\
Group & 0.032 & 1,26 & 0.859 & 0.001 \\
Interaction & 0.442 & 1,26 & 0.512 & 0.017 \\
\hline
\end{tabular}

corticoids previously. No control participant had ever taken glucocorticoids. Antidepressants had been taken previously by one person in each group. One CIJD patient was taking a low-dose tetracyclic antidepressant at the time of the study and none control participant. No included participant was on antihypertensive medication that could have influenced the autonomic nervous system.

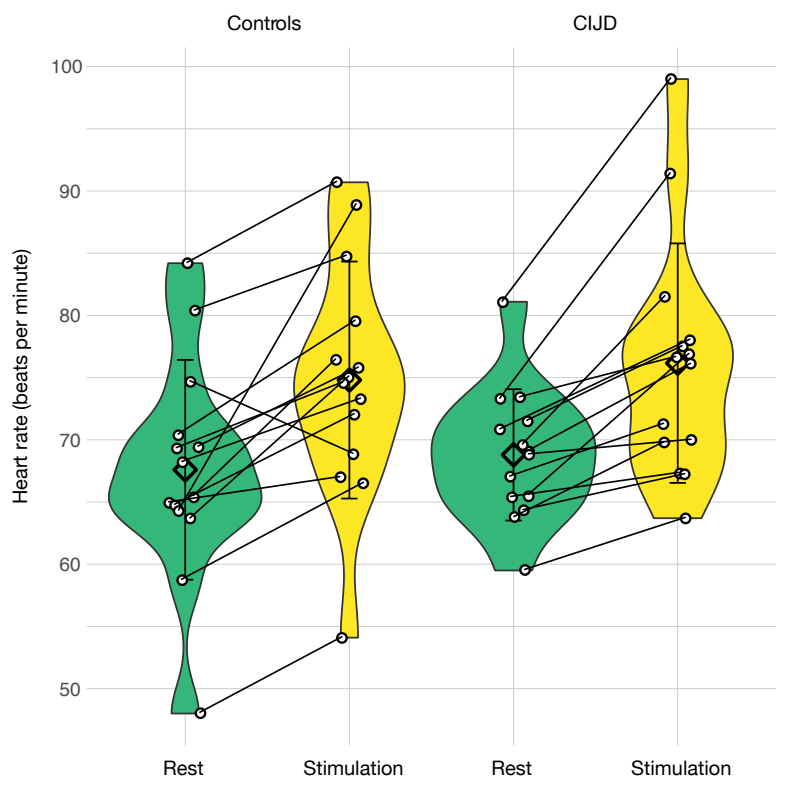

Fig. 2. Violin plot of heart rate at rest and under cognitive stimulation. Controls ( $\mathrm{n}$ $=14$ ) are shown on the left and patients with chronic inflammatory joint diseases (CIJD; $n=14$ ) on the right. Diamonds represent the mean and error bars represent the standard deviation. Green: heart rate $(\mathrm{HR})$ at rest; yellow: heart rate under cognitive stimulation. The ANOVA with repeated measurement showed an increase of HR during stimulation within both groups (condition: $p<0.001, \eta^{2}=0.612$ ). However, there were no differences in $\mathrm{HR}$ at rest and under stimulation between matched groups (group: $\mathrm{p}=0.673, \eta^{2}=0.007$ ).

Heart rate and HRV. Table 2 displays the results of the repeated measurements' ANOVA for HR, SDNN, LF, and HF of the patient and control groups at rest and during cognitive stimulation. In both, controls and patients with CIJD, cognitive stimulation resulted in a significant increase in HR (C: + $10.68 \%$, CIJD: $+10.73 \%$ ) with a large effect size and SDNN 

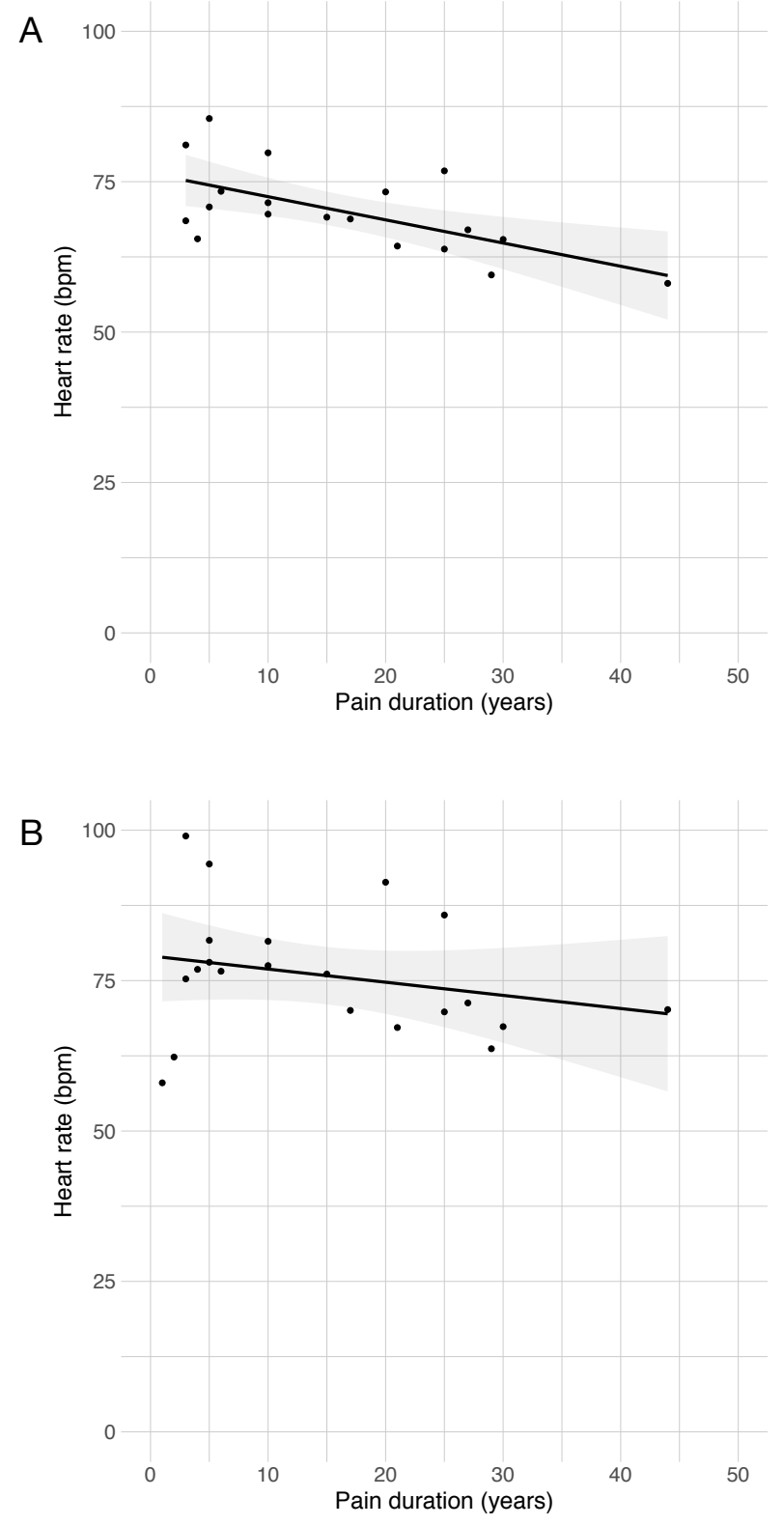

Fig. 3. Correlation between heart rate and pain duration in patients with CIJD. A) Pearson's correlation indicates a strong significant association between heart rate at rest and pain duration $(p=0.004, r=-0.627)$. B) No significant correlation between heart rate under stimulation and pain duration $(p=0.277, r=-0.249)$. The gray area is the $95 \%$ confidence interval with the regression line displayed.

$(\mathrm{C}:+6.11 \%$; CIJD: $+8.08 \%)$ with a medium effect size. Importantly, there were no significant effects between groups. Furthermore, the LF increase in CIJD patients (C: $+2.23 \%$, CIJD: $+12.26 \%$ ) was significant with a medium effect size and no differences in the HF parameters between groups. CIJD patients demonstrated a significant negative correlation between heart rate at rest and pain duration $(\mathrm{r}=-0.627, \mathrm{p}=$ 0.004 , Figure 3A). During cognitive stimulation, there was no correlation between the heart rate and pain duration $(\mathrm{r}=$ $-0.249, p=0.277$, Figure 3B). There were no correlations between SDNN, LF, or HF and pain duration, neither at rest nor during cognitive stimulation.
Table 3. Linear regression model for CIJD patients with heart rate at rest as dependent variable

\begin{tabular}{llllll}
\hline Predictors & Coefficient & SE & Beta & T & p \\
\hline Constant & 65.33 & 21.95 & & 2.98 & $0.009^{*}$ \\
Pain duration & -0.42 & 0.12 & -0.56 & -3.66 & $0.002^{*}$ \\
Sex & 6.57 & 2.75 & 0.34 & 2.39 & $0.030^{*}$ \\
Left Insula & 11919.12 & 4816.80 & 0.38 & 2.47 & $0.026^{*}$ \\
Right Insula & -10209.36 & 3937.89 & -0.45 & -2.59 & $0.020^{*}$ \\
\hline${ }^{*} p<0.05$ & & & & &
\end{tabular}

Structural imaging. Employing independent t-tests of insula volume with eTIV normalization $(C=22$, CIJD $=22)$, we found no significant differences between the matched groups in the left $(\mathrm{t}(42)=0.214, \mathrm{p}=0.832)$ and the right insula $(\mathrm{t}(42)=0.165, \mathrm{p}=0.870)$.

The general linear regression model including group $(\mathrm{C}=18$, CIJD = 19) and HR as dependent variables showed no significant differences $\left(\mathrm{p}=0.349\right.$, adjusted $\left.\mathrm{R}^{2}=0.022\right)$. The SDNN model including group, age, sex, and insula volume in both hemispheres showed significant results and explained $23 \%$ of the variability $(p=0.022)$. However, the only relevant variable was sex. The second subgroup regression model $(n=19)$ was used to predict HR with pain duration, sex, and volume of the insular cortices in both hemispheres. The subgroup regression explained $66 \%$ of the $\mathrm{HR}$ and $39 \%$ for the SDNN variability (HR: $p<0.001$, SDNN: $p=0.021)$. Tables 3 and 4 show the significant results of both regression analyses.

Table 4. Linear regression model for CIJD patients with SDNN at rest as dependent variable

\begin{tabular}{llllll}
\hline Predictors & Coefficient & SE & Beta & T & p \\
\hline Constant & 2.33 & 0.64 & & 3.66 & $0.002^{*}$ \\
Pain duration & 0.01 & 0.003 & 0.37 & 1.79 & 0.093 \\
Sex & -0.28 & 0.08 & -0.66 & -3.52 & $0.003^{*}$ \\
Left Insula & -149.14 & 139.82 & -0.22 & -1.07 & 0.303 \\
Right Insula & -1.26 & 114.31 & -0.003 & -0.01 & 0.991 \\
\hline${ }^{*} p<0.05$ & & & & &
\end{tabular}

\section{Discussion}

Our results provide evidence for an inverse correlation between HR and pain duration in patients with chronic inflammatory joint disease at rest. Moreover, both healthy volunteers and patients with CIJD showed a similar adequate cardiovascular response during cognitive stimulation (serial subtraction). Finally, our data suggest that structural changes in the insular cortices are related to changes in heart rate in CIJD patients.

Heart rate and heart rate variability. The present data provide evidence for a negative association between $H R$ and pain duration with a large effect size $(r=-0.627)$ at rest. To the best of our knowledge, this is the first report of an association between resting HR and pain duration in patients with CIJD. This finding may be explained by an increase in the parasympathetic or a decrease in the sympathetic outflow of the ANS [78]. In an animal model of chronic stress, a decrease of HR was found after stressing mice on a shaking platform for 7 days. This study demonstrated an initial increase of HR in 
response to the stressor, followed by a transition to lower heart rates during the experiment [10]. As part of an adaptation mechanism under longer-lasting stressful or nociceptive stimulations, increased activity of the parasympathetic branch of the ANS could act as a protective factor, maintaining cardiac adaptability for as long as possible [62].

In humans, studies on HR in chronic pain caused by CIJD provided mixed results. For example, Rensburg and colleagues determined a significantly higher basal HR in women with RA [36], whereas Louthrenoo et al. used a non-invasive cardiovascular reflex test (deep breathing) and found no significant difference in maximum and minimum HR in patients with RA compared with healthy controls [43]. The difference between the maximum and minimum HR, however, was significantly smaller in patients with RA compared to controls. Both studies included RA patients with a relatively short mean disease duration of $4.26 \pm 1.2$ years [36] and $5.1 \pm 3.6$ years [43]. By contrast, disease duration in our study was between 1 and 44 years (median: 10 years). It is important to note that there was no significant association between HR under stimulation and pain duration (Figure 3B), suggesting that autonomic control remains intact even in later stages of CIJD.

Similarly, studies on heart rate variability in patients with chronic pain are inconclusive and the selected HRV parameters differ [5]. For example, in 2006, Anichkov and colleagues detected significantly lower HRV in 23 female RA patients compared to matched healthy controls [7]. Contrarily, Vleck and colleagues did not find changes in HRV at rest and under light physical stress in a comparable group (22 female RA patients vs. 15 matched healthy controls) [79].

In our study, we not only measured HR and HRV at rest but also during cognitive stimulation. As expected, HR increased in healthy controls during serial subtraction [6]. Interestingly, our CIJD patients demonstrated a similar increase in HR. So far the most frequently described response to acute stimulation is an HR and LF band increase and a HRV and HF band decrease. This is thought to reflect either a reduction in the parasympathetic or a rise in the sympathetic outflow [38; 78]. In our study, both groups reacted with an increase in HR and SDNN. The CIJD group therefore still seemed to have good cardiac adaptability and reacted appropriately to the stressor. However, only the CIJD reacted with a significant increase in the LF band to cognitive stimulation. The increased LF value in CIJD patients might represent a modified baroreflex [31]. Autonomic imbalance is associated with increased cardiovascular morbidity and mortality [77]. However, to assess the baroreflex more thoroughly was beyond the scope of this study.

Volumes of the left and right insular cortices. Processing of nociceptive input is a highly complex function of the brain, involving multiple brain regions as well as excitatory and inhibitory mechanisms. Structural MRI studies in chronic pain patients have been inconclusive so far with some showing increased and others, decreased cerebral gray matter density [64; 67]. For this study, we performed an automated analysis of the volumes of the left and right insular cortex using FreeSurfer. In a multiple linear regression model including only CIJD patients with HR at rest as a dependent variable, we found a significant positive relationship with the volume of the left insular cortex. Moreover, we found a significant negative relationship between HR at rest and the volume of the right insula (Table 3).

There is still much debate about the lateralization of insular functions, including autonomic control [50; 55]. A widely acknowledged model of insular lateralization proposes that the right insula predominantly activates sympathetic outflow while the left insula mainly initiates parasympathetic outflow $[19 ; 48]$. This model is supported by studies performing unilateral inactivation of the cerebral hemispheres by intracarotid amobarbital procedure [35] and using electrical stimulation of the insulae during epilepsy surgery [51]. Of major clinical importance is the fact that right insular lesions are particularly associated with the development of cardiac arrhythmias $[2 ; 16 ; 18]$. However, this model does not explain all available data and may thus be oversimplified. A recent intraoperative stimulation study did not find differences in HR response between the left and right insula, but between the anterior and posterior parts of both insulae. Left or right posterior insular cortical stimulation predominantly induced tachycardia [15].

Given these controversial findings, the pathomechanistic implications of our results are difficult to assess. Further studies on larger patient populations are needed to replicate these results and to determine if changes in insular volumes might be associated with the increased cardiovascular morbidity and mortality observed in CIJD patients.

ANS changes may not only be caused by central but also by peripheral mechanisms. There is evidence that CIJD can affect afferent peripheral sensory nerves, which in turn lead to sensitization to sympathetic influences [57]. This notion is supported here by a significant increase in sympathetic outflow during stimulation, which could indicate an increased efferent response to the sympathetic nervous system.

Limitations and future research. One of the major strengths of this study is the matching of patients and controls on an individual basis. Further, this study examined diseases that are based on similar pathophysiology. Nevertheless, further research and longitudinal studies are needed to investigate the influence of chronic pain in patients with rheumatic diseases. There is some evidence that changes in brain areas caused by chronic pain were reversible after pain relief [32]. It is possible that some of the morphological brain changes were diminished due to the plasticity of the brain under consistent pain therapy and pain relief or even occurred in other regions that were not examined in this study. It thus would be interesting to follow newly diagnosed patients with rheumatic diseases before and after drug treatment in a long-term study to compare heart and brain modifications.

Using only cognitive instead of physical stimulation can be critical. This study chose mental stimulation due to its relevance to everyday life. Further, a focus on physical stimulations would have required movements, e.g. handgrip exercise, which in turn would be a risk for creating MRI and pulse data 
artifacts [42; 52]. In a future study, it would be interesting to examine whether the CIJD group would reach a limit of their heart adaptability earlier than the control group under physical stimulation. Finally, our group size corresponds to other studies that dealt with similar questions or methods $[8 ; 30$; $41 ; 80]$.

Conclusion. In summary, our results suggest that HR changes are related to pain duration in CIJD patients. Further, our data established the possibility of insular cortex involvement in the process of ANS changes.

\section{Details of authors' contributions}

Study design, ethical approval, and application for funds: CB, PS, MS.

Patient recruitment and data collection: MS, LB.

Data analysis: LB, CB, PS.

Writing manuscript: LB.

Assisting in the review, editing, and writing of the manuscript: $\mathrm{CB}$, PS, MS.

\section{Acknowledgements}

The authors wish to acknowledge the rheumatological clinic of Dr. Markus Voglau, Oldenburg, for the great cooperation in patient recruitment. We thank Katharina Grote and Gülsen Yanç for assisting with MRI data acquisition. We also thank Vivian Baker for editing the final manuscript. The study was supported by the Neuroimaging Unit, University of Oldenburg, funded by grants from the German Research Foundation (DFG; 3T MRI INST 184/152-1 FUGG and MEG INST 184/148-1 FUGG).

\section{References}

[1] Heart rate variability. Standards of measurement, physiological interpretation, and clinical use. Task Force of the European Society of Cardiology and the North American Society of Pacing and Electrophysiology. European Heart Journal 1996;17(3):354-381.

[2] Abboud H, Berroir S, Labreuche J, Orjuela K, Amarenco P. Insular involvement in brain infarction increases risk for cardiac arrhythmia and death. Annals of Neurology 2006;59(4):691-699.

[3] Aboyans V, Ricco J-B, Bartelink M-LE, Björck M, Brodmann M, Cohnert T, Collet J-P, Czerny M, De Carlo M, Debus S. 2017 ESC Guidelines on the Diagnosis and Treatment of Peripheral Arterial Diseases, in collaboration with the European Society for Vascular Surgery (ESVS) Document covering atherosclerotic disease of extracranial carotid and vertebral, mesenteric, renal, upper and lower extremity arteries Endorsed by: the European Stroke Organization (ESO) The Task Force for the Diagnosis and Treatment of Peripheral Arterial Diseases of the European Society of Cardiology (ESC) and of the European Society for Vascular Surgery (ESVS). European Heart Journal 2018;39(9):763-816.

[4] Ackermann H, Riecker A. The contribution(s) of the insula to speech production: a review of the clinical and functional imaging literature. Brain Structure and Function 2010;214(5-6):419-433. [5] Adlan AM, Lip GY, Paton JF, Kitas GD, Fisher JP. Autonomic function and rheumatoid arthritis: a systematic review. Seminars in Arthritis and Rheumatism 2014;44(3):283-304.
[6] Allen TM, Struemph KL, Toledo-Tamula MA, Wolters PL, Baldwin A, Widemann B, Martin S. The Relationship Between Heart Rate Variability, Psychological Flexibility, and Pain in Neurofibromatosis Type 1. Pain Practice 2018;18(8):969-978.

[7] Anichkov DA, Shostak NA, Ivanov DS. Heart rate variability is related to disease activity and smoking in rheumatoid arthritis patients. International Journal of Clinical Practice 2007;61(5):777783.

[8] Apkarian AV, Sosa Y, Sonty S, Levy RM, Harden RN, Parrish TB, Gitelman DR. Chronic back pain is associated with decreased prefrontal and thalamic gray matter density. Journal of Neuroscience 2004;24(46):10410-10415.

[9] Bantel C, Trapp S. The role of the autonomic nervous system in acute surgical pain processing - what do we know? Anaesthesia 2011;66(7):541-544.

[10] Bernatova I, Key MP, Lucot JB, Morris M. Circadian differences in stress-induced pressor reactivity in mice. Hypertension 2002;40(5):768-773.

[11] Berntson GG, Stowell JR. ECG artifacts and heart period variability: don't miss a beat! Psychophysiology 1998;35(1):127-132.

[12] Breivik H, Borchgrevink P, Allen S, Rosseland L, Romundstad L, Hals EB, Kvarstein G, Stubhaug A. Assessment of pain. British Journal of Anaesthesia 2008;101(1):17-24.

[13] Buckner RL, Head D, Parker J, Fotenos AF, Marcus D, Morris JC, Snyder AZ. A unified approach for morphometric and functional data analysis in young, old, and demented adults using automated atlas-based head size normalization: reliability and validation against manual measurement of total intracranial volume. NeuroImage 2004;23(2):724-738.

[14] Cavalli G, Favalli EG. Cardiovascular disease in patients with rheumatoid arthritis: impact of classic and disease-specific risk factors. Annals of Translational Medicine 2018;6(Suppl 1):S82.

[15] Chouchou F, Mauguière F, Vallayer $\mathrm{O}$, Catenoix H, Isnard J, Montavont A, Jung J, Pichot V, Rheims S, Mazzola L. How the insula speaks to the heart: Cardiac responses to insular stimulation in humans. Human Brain Mapping 2019;40(9):2611-2622.

[16] Christensen H, Boysen G, Christensen AF, Johannesen HH. Insular lesions, ECG abnormalities, and outcome in acute stroke. Journal of Neurology, Neurosurgery, and Psychiatry 2005;76(2):269271.

[17] Cohen J. Statistical power analysis for the behavioural sciences. Erlbaum. Hillsdale, NJ 1988.

[18] Colivicchi F, Bassi A, Santini M, Caltagirone C. Cardiac autonomic derangement and arrhythmias in right-sided stroke with insular involvement. Stroke 2004;35(9):2094-2098.

[19] Craig A. Forebrain emotional asymmetry: a neuroanatomical basis? Trends in Cognitive Sciences 2005;9(12):566-571.

[20] Dale AM, Fischl B, Sereno MI. Cortical surface-based analysis. I. Segmentation and surface reconstruction. NeuroImage 1999;9(2):179-194.

[21] Desikan RS, Ségonne F, Fischl B, Quinn BT, Dickerson BC, Blacker D, Buckner RL, Dale AM, Maguire RP, Hyman BT. An automated labeling system for subdividing the human cerebral cortex on MRI scans into gyral based regions of interest. NeuroImage 2006;31(3):968-980.

[22] England BR, Thiele GM, Anderson DR, Mikuls TR. Increased cardiovascular risk in rheumatoid arthritis: mechanisms and implications. British Medical Journal 2018;361:k1036.

[23] Evrard HC. The Organization of the Primate Insular Cortex. Frontiers in Neuroanatomy 2019;13(43).

[24] Evrengul H, Dursunoglu D, Cobankara V, Polat B, Seleci D, Kabukcu S, Kaftan A, Semiz E, Kilic M. Heart rate variability 
in patients with rheumatoid arthritis. Rheumatology International 2004;24(4):198-202.

[25] Fischl B, Liu A, Dale AM. Automated manifold surgery: constructing geometrically accurate and topologically correct models of the human cerebral cortex. IEEE Transactions on Medical Imaging 2001;20(1):70-80.

[26] Fischl B, Salat DH, Busa E, Albert M, Dieterich M, Haselgrove C, van der Kouwe A, Killiany R, Kennedy D, Klaveness S, Montillo A, Makris N, Rosen B, Dale AM. Whole brain segmentation: automated labeling of neuroanatomical structures in the human brain. Neuron 2002;33(3):341-355.

[27] Fischl B, Salat DH, van der Kouwe AJ, Makris N, Segonne F, Quinn BT, Dale AM. Sequence-independent segmentation of magnetic resonance images. NeuroImage 2004;23 Suppl 1:S69-84.

[28] Fischl B, Van Der Kouwe A, Destrieux C, Halgren E, Ségonne F, Salat DH, Busa E, Seidman LJ, Goldstein J, Kennedy D. Automatically parcellating the human cerebral cortex. Cerebral Cortex 2004;14(1):11-22.

[29] Frot M, Faillenot I, Mauguière F. Processing of nociceptive input from posterior to anterior insula in humans. Human Brain Mapping 2014;35(11):5486-5499.

[30] Geha PY, Baliki MN, Harden RN, Bauer WR, Parrish TB, Apkarian AV. The brain in chronic CRPS pain: abnormal gray-white matter interactions in emotional and autonomic regions. Neuron 2008;60(4):570-581.

[31] Goldstein DS, Bentho O, Park MY, Sharabi Y. Low-frequency power of heart rate variability is not a measure of cardiac sympathetic tone but may be a measure of modulation of cardiac autonomic outflows by baroreflexes. Experimental Physiology 2011;96(12):1255-1261.

[32] Gwilym SE, Filippini N, Douaud G, Carr AJ, Tracey I. Thalamic atrophy associated with painful osteoarthritis of the hip is reversible after arthroplasty: A longitudinal voxel-based morphometric study. Arthritis \& Rheumatism 2010;62(10):2930-2940.

[33] Han X, Jovicich J, Salat D, van der Kouwe A, Quinn B, Czanner S, Busa E, Pacheco J, Albert M, Killiany R, Maguire P, Rosas D, Makris N, Dale A, Dickerson B, Fischl B. Reliability of MRIderived measurements of human cerebral cortical thickness: the effects of field strength, scanner upgrade and manufacturer. NeuroImage 2006;32(1):180-194.

[34] Hilz MJ, Dütsch M. Quantitative studies of autonomic function. Muscle Nerve 2006;33(1):6-20.

[35] Hilz MJ, Dütsch M, Perrine K, Nelson PK, Rauhut U, Devinsky O. Hemispheric influence on autonomic modulation and baroreflex sensitivity. Annals of Neurology 2001;49(5):575-584.

[36] Janse van Rensburg DC, Ker JA, Grant CC, Fletcher L. Autonomic impairment in rheumatoid arthritis. International Journal of Rheumatic Diseases 2012;15(4):419-426.

[37] Kazis LE, Meenan RF, Anderson JJ. Pain in the rheumatic diseases. Arthritis \& Rheumatology 1983;26(8):1017-1022.

[38] Kim HG, Cheon EJ, Bai DS, Lee YH, Koo BH. Stress and Heart Rate Variability: A Meta-Analysis and Review of the Literature. Psychiatry Investigation 2018;15(3):235-245.

[39] Kirschbaum C, Pirke K-M, Hellhammer DH. The 'Trier Social Stress Test'-a tool for investigating psychobiological stress responses in a laboratory setting. Neuropsychobiology 1993;28(12):76-81.

[40] Kraynak TE, Marsland AL, Wager TD, Gianaros PJ. Functional neuroanatomy of peripheral inflammatory physiology: A metaanalysis of human neuroimaging studies. Neuroscience and Biobehavioral Reviews 2018;94:76-92.

[41] Kuchinad A, Schweinhardt P, Seminowicz DA, Wood PB,
Chizh BA, Bushnell MC. Accelerated brain gray matter loss in fibromyalgia patients: premature aging of the brain? Journal of Neuroscience 2007;27(15):4004-4007.

[42] Lalande S, Sawicki CP, Baker JR, Shoemaker JK. Effect of age on the hemodynamic and sympathetic responses at the onset of isometric handgrip exercise. Journal of Applied Physiology 2014;116(2):222-227.

[43] Louthrenoo W, Ruttanaumpawan P, Aramrattana A, Sukitawut W. Cardiovascular autonomic nervous system dysfunction in patients with rheumatoid arthritis and systemic lupus erythematosus. Quarterly Journal of Medicine 1999;92(2):97-102.

[44] Masoud S, Lim PB, Kitas GD, Panoulas V. Sudden cardiac death in patients with rheumatoid arthritis. World Journal of Cardiology 2017;9(7):562-573.

[45] Mathalon DH, Sullivan EV, Rawles JM, Pfefferbaum A. Correction for head size in brain-imaging measurements. Psychiatry Research 1993;50(2):121-139.

[46] Montano N, Ruscone TG, Porta A, Lombardi F, Pagani M, Malliani A. Power spectrum analysis of heart rate variability to assess the changes in sympathovagal balance during graded orthostatic tilt. Circulation 1994;90(4):1826-1831.

[47] Nagai M, Dote K, Kato M, Sasaki S. The Right Insular Cortex: A Critical Region for Modulating the Parasympathetic Activity? Journal of Neurosurgical Anesthesiology 2016;28(2):176.

[48] Nagai M, Hoshide S, Kario K. The insular cortex and cardiovascular system: a new insight into the brain-heart axis. Journal of the American Society of Hypertension 2010;4(4):174-182.

[49] Niskanen JP, Tarvainen MP, Ranta-Aho PO, Karjalainen PA. Software for advanced HRV analysis. Computer Methods and Programs in Biomedicine 2004;76(1):73-81.

[50] Oppenheimer S. Cerebrogenic cardiac arrhythmias: Cortical lateralization and clinical significance. Clinical Autonomic Research 2006;16(1):6-11.

[51] Oppenheimer SM, Gelb A, Girvin JP, Hachinski VC. Cardiovascular effects of human insular cortex stimulation. Neurology 1992;42(9):1727-1732.

[52] Perini R, Veicsteinas A. Heart rate variability and autonomic activity at rest and during exercise in various physiological conditions. European Journal of Applied Physiology 2003;90(3-4):317325.

[53] Ponikowski P, Voors AA, Anker SD, Bueno H, Cleland JG, Coats AJ, Falk V, Gonzalez-Juanatey JR, Harjola V-P, Jankowska EA. 2016 ESC Guidelines for the diagnosis and treatment of acute and chronic heart failure: The Task Force for the diagnosis and treatment of acute and chronic heart failure of the European Society of Cardiology (ESC) Developed with the special contribution of the Heart Failure Association (HFA) of the ESC. European Heart Journal 2016;37(27):2129-2200.

[54] Reyes del Paso GA, Langewitz W, Mulder LJ, van Roon A, Duschek S. The utility of low frequency heart rate variability as an index of sympathetic cardiac tone: a review with emphasis on a reanalysis of previous studies. Psychophysiology 2013;50(5):477487.

[55] Ruiz Vargas E, Sörös P, Shoemaker JK, Hachinski V. Human cerebral circuitry related to cardiac control: A neuroimaging metaanalysis. Annals of Neurology 2016;79(5):709-716.

[56] Ruscheweyh R, Deppe M, Lohmann H, Stehling C, Floel A, Ringelstein EB, Knecht $S$. Pain is associated with regional grey matter reduction in the general population. Pain 2011;152(4):904-911.

[57] Sato J, Suzuki S, Iseki T, Kumazawa T. Adrenergic excitation of cutaneous nociceptors in chronically inflamed rats. Neuroscience Letters 1993;164(1-2):225-228. 
[58] Schmidt-Wilcke T, Gänssbauer S, Neuner T, Bogdahn U, May A. Subtle grey matter changes between migraine patients and healthy controls. Cephalalgia 2008;28(1):1-4.

[59] Schmidt-Wilcke T, Leinisch E, Straube A, Kampfe N, Draganski B, Diener HC, Bogdahn U, May A. Gray matter decrease in patients with chronic tension type headache. Neurology 2005;65(9):1483-1486.

[60] Segonne F, Dale AM, Busa E, Glessner M, Salat D, Hahn HK, Fischl B. A hybrid approach to the skull stripping problem in MRI. NeuroImage 2004;22(3):1060-1075.

[61] Segonne F, Pacheco J, Fischl B. Geometrically accurate topology-correction of cortical surfaces using nonseparating loops. IEEE Transactions on Medical Imaging 2007;26(4):518-529.

[62] Selye H. Stress without Distress. In: Serban G. (ed) Psychopathology of Human Adaptation. Springer, Boston, MA 1976. pp. 137-146.

[63] Shaffer F, Ginsberg JP. An Overview of Heart Rate Variability Metrics and Norms. Frontiers in Public Health 2017;5:258.

[64] Shi H, Yuan C, Dai Z, Ma H, Sheng L. Gray matter abnormalities associated with fibromyalgia: a meta-analysis of voxelbased morphometric studies. Seminars in Arthritis and Rheumatism 2016;46(3):330-337.

[65] Shu L, Wang Y. Highlights in basic autonomic neuroscience: insular cortex injury leads to cardiovascular dysfunction. Autonomic Neuroscience: Basic \& Clinical 2014;185:1-4.

[66] Sled JG, Zijdenbos AP, Evans AC. A nonparametric method for automatic correction of intensity nonuniformity in MRI data. IEEE Transactions on Medical Imaging 1998;17(1):87-97.

[67] Smallwood RF, Laird AR, Ramage AE, Parkinson AL, Lewis J, Clauw DJ, Williams DA, Schmidt-Wilcke T, Farrell MJ, Eickhoff SB, Robin DA. Structural brain anomalies and chronic pain: a quantitative meta-analysis of gray matter volume. Pain 2013;14(7):663675.

[68] Solomon DH, Karlson EW, Rimm EB, Cannuscio CC, Mandl LA, Manson JE, Stampfer MJ, Curhan GC. Cardiovascular morbidity and mortality in women diagnosed with rheumatoid arthritis. Circulation 2003;107(9):1303-1307.

[69] Sörös P, Bantel C. Chronic noncancer pain is not associated with accelerated brain aging as assessed by structural magnetic resonance imaging in patients treated in specialized outpatient clinics. Pain 2020;161(3):641-650.

[70] Sörös P, Hachinski V. Cardiovascular and neurological causes of sudden death after ischaemic stroke. Lancet Neurology 2012;11(2):179-188.

[71] Sörös P, Inamoto Y, Martin RE. Functional brain imaging of swallowing: an activation likelihood estimation meta-analysis. Human Brain Mapping 2009;30(8):2426-2439.

[72] Sörös P, Lalone E, Smith R, Stevens T, Theurer J, Menon RS, Martin RE. Functional MRI of oropharyngeal air-pulse stimulation. Neuroscience 2008;153(4):1300-1308.

[73] Sörös P, Marmurek J, Tam F, Baker N, Staines WR, Graham SJ. Functional MRI of working memory and selective attention in vibrotactile frequency discrimination. BMC Neuroscience 2007;8:48. [74] Spindler M, Koch K, Borisov E, Ozyurt J, Sörös P, Thiel C, Bantel C. The Influence of Chronic Pain and Cognitive Function on Spatial-Numerical Processing. Frontiers in Behavioral Neuroscience 2018;12:165.

[75] Tarvainen MP, Niskanen JP, Lipponen JA, Ranta-Aho PO, Karjalainen PA. Kubios HRV - heart rate variability analysis software. Computer Methods and Programs in Biomedicine 2014;113(1):210220.

[76] Thayer JF, Ahs F, Fredrikson M, Sollers JJ, 3rd, Wager TD. A meta-analysis of heart rate variability and neuroimaging studies: implications for heart rate variability as a marker of stress and health. Neuroscience and Biobehavioral Reviews 2012;36(2):747-756.

[77] Thayer JF, Yamamoto SS, Brosschot JF. The relationship of autonomic imbalance, heart rate variability and cardiovascular disease risk factors. International Journal of Cardiology 2010;141(2):122131.

[78] Tracy LM, Ioannou L, Baker KS, Gibson SJ, GeorgiouKaristianis N, Giummarra MJJP. Meta-analytic evidence for decreased heart rate variability in chronic pain implicating parasympathetic nervous system dysregulation. Pain 2016;157(1):7-29.

[79] Vlcek M, Rovensky J, Eisenhofer G, Radikova Z, Penesova A, Kerlik J, Imrich R. Autonomic nervous system function in rheumatoid arthritis. Cellular and Molecular Neurobiology 2012;32(5):897901.

[80] Wartolowska K, Hough MG, Jenkinson M, Andersson J, Wordsworth BP, Tracey I. Structural changes of the brain in rheumatoid arthritis. Arthritis \& Rheumatology 2012;64(2):371-379.

[81] Wolfe F, Mitchell DM, Sibley JT, Fries JF, Bloch DA, Williams CA, Spitz PW, Haga M, Kleinheksel SM, Cathey MA. The mortality of rheumatoid arthritis. Arthritis \& Rheumatology 1994;37(4):481494. 